\title{
Environmental effects on the ionisation of brown dwarf atmospheres
}

\author{
M. I. Rodríguez-Barrera ${ }^{1,2}$, Ch. Helling ${ }^{1,2,3}$, and K. Wood $^{2}$ \\ ${ }^{1}$ Centre for Exoplanet Science, University of St Andrews, UK \\ e-mail: christiane.helling@st-and.ac.uk \\ 2 School of Physics \& Astronomy, University of St. Andrews, St. Andrews KY16 9SS, UK \\ 3 Kapteyn Astronomical Institute, Postbus 800, 9700 AV Groningen, The Netherlands
}

Received 22 January 2018 / Accepted 24 April 2018

\begin{abstract}
Context. Brown dwarfs emit bursts of $\mathrm{H} \alpha$, white-light flares, and show radio flares and quiescent radio emission. They are suggested to form aurorae, similar to planets in the solar system, but much more energetic. All these processes require a source gas with an appropriate degree of ionisation, which, so far, is mostly postulated to be sufficient.

Aims. We aim to demonstrate that the Galactic environment influences atmospheric ionisation, and that it hence amplifies or enables the magnetic coupling of the atmospheres of ultra-cool objects, like brown dwarfs and free-floating planets.

Methods. We build on our previous work on thermal ionisation of ultra-cool atmospheres and explore the effect of environmental high-energy radiation on the degree of ionisation in the atmosphere. We consider the effect of photoionisation by Lyman-continuum radiation in three different environments: in the interstellar radiation field (ISRF), O and B stars in star-forming regions, and in white dwarf companions in binary systems. We apply our Monte Carlo radiation transfer code to investigate the effect of Lyman-continuum photoionisation for prescribed atmosphere structures for very low-mass objects.

Results. The external radiation environment plays an important role for the atmospheric ionisation of very low-mass, ultra-cool objects. Lyman-continuum irradiation greatly increases the level of ionisation in the uppermost atmospheric regions. Our results suggest that a shell of an almost fully ionised atmospheric gas emerges for brown dwarfs in star-forming regions and brown dwarfs in white dwarf binary systems. As a consequence, brown dwarf atmospheres can be magnetically coupled, which is the presumption for chromospheric heating to occur and for aurorae to emerge. First tests for assumed chromosphere-like temperature values suggest that the resulting free-free X-ray luminosities are comparable with those observed from non-accreting brown dwarfs in star-forming regions.
\end{abstract}

Key words. radiation mechanisms: non-thermal - planet-star interactions - stars: atmospheres - brown dwarfs

\section{Introduction}

Radio and X-ray emission from ultra-cool objects has been reported by Berger (2002), Stelzer (2004), Hallinan et al. (2006), Osten et al. (2009), Route \& Wolszczan (2012), Burgasser et al. (2015), Williams et al. (2014) and Kao et al. (2016) for example, and surveys are being conducted (Antonova et al. 2013; Pineda et al. 2016, 2017). The white-flare observations by Schmidt et al. (2016; for ASASSN-16AE) and Gizis et al. (2013; for W1906+40) provide evidence for stellar-type magnetic activity to occur also in brown dwarfs, and hence, Schmidt et al. (2015) and Sorahana et al. (2014) suggested that brown dwarfs have chromospheres. Stelzer (2004) studied the old brown dwarf G569Bab and concluded that coronal emission remains powerful also beyond young ages. The presence of a magnetised atmospheric plasma, including a sufficient number of electrons, is required to allow the formation of a chromosphere/corona through magnetohydrodynamics (MHD) processes such as wave heating, similar to the Sun (Brady \& Arber 2016; Mullan \& MacDonald 2016; Reep \& Russell 2016). Seed electrons are also required to understand aurorae on moon-less brown dwarfs.

Rodríguez-Barrera et al. (2015) showed that thermal ionisation can produce a partially ionised gas in a substantial volume of cool brown dwarfs and giant gas planet atmospheres and a highly ionised gas in the hotter/younger brown dwarfs or M dwarfs. However, for low effective temperatures and low surface gravity, additional mechanisms are required to ionise the upper atmosphere to a degree that radio and X-ray observation become feasible, as was also suggested by Mohanty et al. (2002). Rimmer \& Helling (2013) demonstrated that cosmicray $(\mathrm{CR})$ irradiation significantly enhances the electron fraction compared to thermal ionisation in the upper atmospheres of brown dwarfs, but the local degree of ionisation does not exceed $10^{-7}$ in these low-density regions. Helling et al. (2013) and Rimmer \& Helling (2013) further pointed out that CRs can affect the upper portion of the mineral clouds that form in brown dwarf atmospheres (Helling \& Casewell 2014), an effect that is well established for solar system objects (Helling et al. 2016a). The present paper takes the idea of environmental effects on the ionisation of atmospheres of very low-mass, ultra-cool objects one step further.

Ultra-cool objects, that is, brown dwarfs and free-floating planets, are observed in a great variety of environments. Brown dwarfs and free-floating planets in star-forming regions are exposed to a stronger radiation field than objects that are situated in the interstellar medium (ISM). Star-forming regions host $\mathrm{O}$ and $\mathrm{B}$ stars that produce a substantial fraction of highenergy radiation that may lead to the ionisation of the outer 
atmosphere of brown dwarfs. Sicilia-Aguilar et al. (2008) and Forbrich \& Preibisch (2007) showed that non-accreting brown dwarfs in star-forming regions have X-ray luminosities of the order of $\log _{10} L_{\mathrm{x}} \approx 28\left[\mathrm{erg} \mathrm{s}^{-1}\right.$ ] for $0.5 \ldots 8 \mathrm{KeV}$. Kashyap et al. (2008) presented X-ray observations for planet-host stars, including $\mathrm{M}$ dwarfs and brown dwarfs (M2 ... M8.5) with X-ray luminosities of $\log _{10} L_{\mathrm{x}}=26.36 \ldots 31.22 \mathrm{erg} \mathrm{s}^{-1}$. This X-ray emission is therefore likely to originate from chromospheric activity of the brown dwarfs and low-mass stars.

In this paper we investigate the effect of Lyman-continuum radiation (from photons with energies above the Lyman limit of $E>13.6 \mathrm{eV}$, hence of wavelengths $\lambda<912 \AA$ ) in different Galactic environments on the ionisation of the atmospheric gas of brown dwarfs. We study three different cases: (i) irradiation from the interstellar radiation field (ISRF), (ii) irradiation from $\mathrm{O}$ and $\mathrm{B}$ stars within a star-forming region, and (iii) irradiation from a white dwarf companion. Our interest in case (iii) is supported by the recent finding of atomic line emission from a brown dwarf in the white dwarf and brown dwarf system (WD-BD) binary WD0137-349 (Longstaff et al. 2017). We use the analysis framework from Rodríguez-Barrera et al. (2015) to study the resulting plasma parameters and the possible magnetic coupling of the atmosphere. Our results support the expectation that brown dwarfs form chromospheres, and we make the first suggestions for the X-ray flux possibly emerging from a shell of optically thin, but hot gas that may form the outer part of brown dwarf atmospheres. Our approach is summarised in Sect. 2. We present our results in Sect. 3. Section 4 contains our discussion, and Sect. 5 contains our conclusions.

\section{Approach}

We used DrifT-PHOENIX atmosphere model structures $\left(T_{\text {eff }}=\right.$ $2800 \mathrm{~K}, 2000 \mathrm{~K}, 1000 \mathrm{~K}, \log (g)=3.0,5.0,[\mathrm{M} / \mathrm{H}]=0.0$; Helling et al. 2008a, 2008b; Witte et al. 2009, 2011) as input for Monte Carlo radiative transfer photoionisation calculations. Using global parameters that describe young and old brown dwarfs $\left(T_{\text {eff }}[\mathrm{K}], \log (g)\left[\mathrm{cm} \mathrm{s}^{-2}\right],[\mathrm{M} / \mathrm{H}]\right)$, the model atmosphere simulations DRIFT-PHOENIX provide the local gas properties $\left(T_{\text {gas }}[\mathrm{K}], p_{\text {gas }}[\mathrm{bar}], p_{\mathrm{e}}\right.$ [bar]; Fig. 1). DRIFT-PHOENIX also calculates the detailed cloud structure in ultra-cool atmospheres (Woitke \& Helling 2004; Helling \& Woitke 2006; Helling \& Casewell 2014). For this paper, we used the cloud particle number density, $n_{\mathrm{d}}$ (for more details on clouds, see e.g. Helling et al. 2016b), to determine whether Lyman-continuum photons might reach deep enough into the atmosphere to also be a source for cloud particle ionisation similar to cosmic ray ionisation. This is, however, only a side-line of the present paper.

We evaluated the effect of external Lyman-continuum (LyC) irradiation in three different environments:

(i) ISM. This includes old brown dwarfs and free-floating planes irradiated by the interstellar radiation field (ISRF). We used the ISRF as a lower limit for the Lyman-continuum irradiation causing possible atmospheric ionisation in brown dwarfs.

(ii) Star-forming region. Brown dwarfs reside in environments with a strong radiation field, such as star-forming regions with $\mathrm{O}$ and $\mathrm{B}$ stars. $\mathrm{O}$ stars allow us to explore an upper limit of the irradiation effect that is due to LyC, while B stars are more common members of star-forming regions, as in the Orion OB1 stellar association.

(iii) White dwarf - brown dwarf binaries (WD-BD). Old brown dwarfs can be companions of a white dwarf. As an

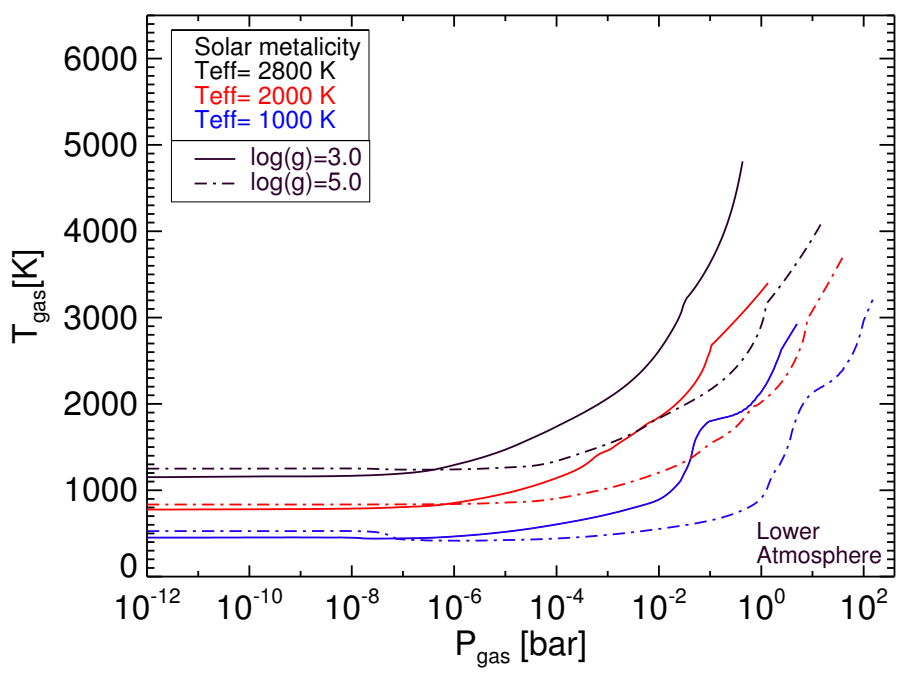

Fig. 1. $\left(T_{\text {gas }}-p_{\text {gas }}\right)$ profile from the DRIFT-PhoENIX model atmosphere simulations. (The $\left(p_{\text {gas }}-z\right)$ correlation can be found in Fig. A.1.) These models are used as input for the Monte Carlo photoionisation calculation. The hottest atmosphere represents a late-M dwarf or young brown dwarf atmosphere. The coolest atmosphere represents a planetary regime atmosphere.

example, we use the binary system WD0837+185, which is composed of a cool white dwarf and an old brown dwarf with an orbital separation of $d=0.006 \mathrm{AU}$ (Casewell et al. $2015)^{1}$. Despite the low occurrence rate of WD-BD binaries of only $0.5 \%$ (Steele et al. 2013), these systems are an important link to giant gas planets since WD-BD pairs are far easier to observe.

\subsection{Photoionisation by Lyman-continuum irradiation}

In an external radiation field originating from an $\mathrm{O}$ or a $\mathrm{B}$ star, a white dwarf, or the ISRF, the radiation energy $E>$ $13.6 \mathrm{eV}$ first dissociates the atmospheric $\mathrm{H}_{2}$, which leaves the gas in the upper atmosphere to be dominated by atomic hydrogen. This is supported by Rimmer \& Helling (2016; their Fig. 9), who showed that $\mathrm{H}_{2}$ is transformed into $\mathrm{H}$ in the uppermost atmospheric layers of an irradiated planet. Lystrup et al. (2008) pointed out that the upper atmosphere of Jupiter is dominated by $\mathrm{H}_{2}$, that $\mathrm{H}_{3}^{+}$has been consistently predicted by models to be a major component of the ionosphere of Jupiter, but that $\mathrm{H}^{+}$predominates at higher altitudes and on the night side. $\mathrm{H}_{3}^{+}$has a short lifetime and therefore quickly decays into atomic hydrogen, but $\mathrm{H}^{+}$is long-lived as it requires three-body electron recombinations or a radiative recombination reaction. Rimmer et al. (2014) showed that the dominating ionic molecule resulting from cosmic-ray triggered ion-neutral chemistry is $\mathrm{NH}_{4}^{+}$, which also leads to $\mathrm{H}_{2}$ dissociation. More work is required to solidify this argument, also to clarify whether super-thermal electrons might be emerging. However, we note that only a very small part $(\sim 0.1 \%)$ of the atmosphere will be ionised, so that the bulk $(\sim 99.9 \%)$ remains $\mathrm{H}_{2}$-dominated. We applied a Monte Carlo radiative transfer (MCRT) to investigate the ionisation of

1 WD0837+185 is a binary system composed of a white dwarf and a low-mass companion with a close orbital separation ( $<3$ AU). Such systems are very rare. The low number of such known systems might be linked to their formation mechanism (Casewell et al. 2013, 2012). WD0837+185 is one of the four known white dwarf-brown dwarf binary systems (Casewell et al. 2015). 
atomic hydrogen by Lyman-continuum photons. The abundance of $\mathrm{H}^{+}$, and therefore the number of electrons originating from Lyman-continuum irradiation, was derived by applying a Monte Carlo photoionisation code (Wood \& Loeb 2000).

The code balances photoionisations with radiative recombinations by the ionisation equilibrium equation in each cell to achieve a new ionisation structure,

$n(H) \int_{v_{o}}^{\infty} \frac{4 \pi J_{v}}{h v} a_{v}(H) \mathrm{d} v=n_{\mathrm{e}} n_{\mathrm{p}} \alpha(H, T)$,

where $n(H)$ is the neutral hydrogen atom (HI) density $\left(\mathrm{cm}^{-3}\right)$, $J_{v}$ is the mean intensity $\left(\mathrm{erg} \mathrm{cm}-2 \mathrm{~s}^{-1} \mathrm{~Hz}^{-1}\right), a_{v}(H)=6.3 \times$ $10^{-18} \mathrm{~cm}^{2}$ is the ionisation cross section for the hydrogen with $E>h v_{\mathrm{o}}(E>13.6 \mathrm{eV}), \alpha(H, T)$ is the recombination coefficient $\left(\mathrm{cm}^{3} \mathrm{~s}^{-1}\right)$, and $n_{\mathrm{e}}$ and $n_{\mathrm{p}}\left(\mathrm{cm}^{-3}\right)$ are the electron and proton densities, respectively. The opacity was updated according to the neutral fraction of hydrogen, and the next radiation transfer iteration was carried out. The Monte Carlo radiation transfer code tracks photon paths and computes the ionisation structure of hydrogen. We used a atmospheric density grid with input from DRIFT-PHOENIX models. It was homogeneously extended onto the 3D MC grid. We therefore used a 3D slab structure with an atmospheric density gradient in order to determine whether a principle effect of LyC irradiation on ultra-cool atmospheres occurred. We are interested in the depth to which external radiation may potentially ionise the atmosphere in addition to cosmic rays, as demonstrated in Rimmer \& Helling (2013).

Input parameters. The Monte Carlo code follows the random walks within the atmosphere grid for both direct ionising photons from the source(s) and diffuse photons produced by radiative recombinations direct to the ground-state of hydrogen. We adopted the same approach as Wood \& Loeb (2000) and assigned all direct stellar photons an energy of $18 \mathrm{eV}$ and diffuse photons an energy of $13.6 \mathrm{eV}$. The hydrogen ionisation cross sections at these energies are $6.3 \times 10^{-18} \mathrm{~cm}^{2}$ and $2.7 \times 10^{-18}$ $\mathrm{cm}^{2}$, respectively. Our simulations only tracked the ionisation of hydrogen, so this two-energy approximation for the direct and diffuse photons is sufficient to determine the depth-dependent ionisation structure. Had we wished to know the detailed depthdependent ionisation structure of an element with multiple ionisation stages, then we would require an input spectrum as described in the Monte Carlo photoionisation code of Wood et al. (2004).

Direct ionising photons that are absorbed by neutral hydrogen in the Monte Carlo simulation are converted into diffuse photons with a probability $\alpha_{1} / \alpha_{A}$, where $\alpha_{1}$ is the hydrogen recombination coefficient direct to the ground-state and $\alpha_{\mathrm{A}}$ is the recombination coefficient to all levels. We adopted a radiative recombination rate $\alpha_{A}=4 \times 10^{-13} \mathrm{~cm}^{3} \mathrm{~s}^{-1}$ and the ratio $\alpha_{1} / \alpha_{A}=0.38$, which is appropriate for photoionised gas at $10^{4} \mathrm{~K}$ (Osterbrock \& Ferland 2006). A key input parameter for the Monte Carlo photoionisation simulations is the flux of LyC photons at $18 \mathrm{eV}(688 \AA)$ reaching the top of the atmospheres from the different sources (Table 1). It is determined by the adopted ionising luminosities and source-atmosphere distances.

We conducted test calculations to determine how different input photon energies (corresponding to different cross-section values) may affect our resulting values for the ionisation penetration depth. We find that for the high densities in the brown dwarf atmospheres we study that the total ionising luminosity is the most important parameter for determining the depth of
Table 1. Ionising luminosities, $Q$, adopted for OB stars in Sternberg et al. (2003), white dwarfs (Hills 1973; Casewell et al. 2012) and the resulting ionising fluxes, $F_{\mathrm{LyC}}$, reaching the top of the irradiated atmosphere for the quoted separations.

\begin{tabular}{lcccl}
\hline \hline Source & $\begin{array}{c}T_{\text {eff }} \\
{[\mathrm{K}]}\end{array}$ & $\begin{array}{c}Q \\
{[\text { photons s }}\end{array}$ & $d$ & $\begin{array}{c}F_{\mathrm{LyC}} \\
\text { [photons s }\end{array} \mathrm{cm}^{-2}$ ] \\
\hline O3 & 51230 & $7.41 \times 10^{49}$ & $0.5 \mathrm{pc}$ & $2.5 \times 10^{13}$ \\
B0 & 33340 & $1.05 \times 10^{48}$ & $0.5 \mathrm{pc}$ & $3.5 \times 10^{10}$ \\
WD & 14748 & $2.75 \times 10^{41}$ & $0.006 \mathrm{AU}$ & $2.7 \times 10^{18}$ \\
ISRF & - & - & - & $3.0 \times 10^{7}$ \\
\hline
\end{tabular}

Notes. For the ISRF, the ionising flux is taken from Reynolds (1984).

photoionisation and that the energy values adopted for the direct and diffuse photons have a negligible effect.

\subsection{Free-free emission}

The ionised hydrogen gas in the upper atmospheres of irradiated brown dwarfs may emit free-free radiation (thermal Bremsstrahlung) if the temperature is high enough. If the gas is optically thin, the resulting luminosity is calculated by

$L_{\mathrm{tot}}^{\mathrm{ff}}=4 \pi \int_{R}^{z_{\max }}\left(R+\left(z_{\max }-z\right)\right)^{2} \epsilon_{v}^{\mathrm{ff}}(z) \mathrm{d} z$,

with $R(\mathrm{~cm})$ the radius of the irradiated object, and $z_{\max }(\mathrm{cm})$ the maximum value of the vertical geometrical extension of the atmosphere. The volume emissivity or the total free-free emission power that a gas can emit per unit of volume, per solid angle, and per unit of frequency, $\epsilon_{v}^{\mathrm{ff}}(z)\left(\mathrm{erg} \mathrm{cm}^{-3} \mathrm{~s}^{-1}\right)$, of an optically thin gas integrated over a range of energy (Osterbrock \& Ferland 2006) is given by the equation

$\epsilon^{\mathrm{ff}}=5.44 \times 10^{-39} T_{\mathrm{em}}^{1 / 2} n_{\mathrm{e}} n_{\mathrm{i}} \overline{\mathrm{g}}_{B} \frac{1}{h} \int_{h v_{1}}^{h v_{2}} \mathrm{e}^{-\frac{h \nu}{k_{\mathrm{B}} T}} \mathrm{~d}(h v)$.

The electron density, $n_{\mathrm{e}}\left(\mathrm{cm}^{-3}\right)$ results from the Monte Carlo calculation. $T_{\mathrm{em}}[\mathrm{K}]$ is the temperature of the emitting gas, $k_{\mathrm{B}}$ $\left(\mathrm{eV} \mathrm{K}^{-1}\right)$ is the Boltzmann constant, $h(\mathrm{eV} \mathrm{s})$ is the Planck constant, and $\overline{\mathrm{g}}_{\mathrm{B}}=1.2$ is the Gaunt factor for hydrogen plasma (Osterbrock \& Ferland 2006). The integration boundaries $h v_{1}=$ $0.5 \mathrm{KeV}$ and $h v_{2}=8 \mathrm{KeV}$ correspond to the X-ray range of the electromagnetic spectra.

This simple approach allowed us to test first estimates for the X-ray luminosity that an irradiated brown dwarf may emit. No model for the formation of a brown dwarf chromosphere/corona is available to consistently derive the X-ray luminosity. Mullan \& MacDonald (2016) suggested that Alfvén waves are an efficient mechanism for heating the corona of M dwarfs. They modelled a mechanical energy flux (Alfvén waves) to explain observed X-ray fluxes from the corona of M dwarfs. To the authors' knowledge, only Wedemeyer et al. (2013) performed a consistent modelling of a chromosphere for an $M$ dwarf. We therefore followed an approach similar to Feigelson et al. (2003), Fuhrmeister et al. (2010) and Schmidt et al. (2015). Fuhrmeister et al. (2005) constructed a semi-empirical chromosphere model for quiescent mid-M dwarfs following examples from the solar community (see references in their paper). Fuhrmeister et al. (2010) applied this model to the flaring M5.5 star CN Leo. A linear temperature rise in transition region and chromosphere was modelled, and the gradient was adjusted. The top of the chromosphere had a prescribed temperature 
of $8000 \mathrm{~K}$ (quiescent chromosphere) or $8500 \mathrm{~K}$ (flaring chromosphere). Higher temperatures were considered in order to represent the heating by flares. Schmidt et al. (2015) followed this approach by replacing the temperature in the outer atmosphere with a chromospheric temperature structure consisting of two components (their Fig. 8). The chromospheric temperature rise, the chromosphere break, and the start of the transition region were treated as free parameters, because little is known about the formation of chromospheres on brown dwarfs and late-M dwarfs. They calculated activity strengths for their set of 11820 late-M and brown dwarfs. Here, the 1D model consists of an underlying (cloudforming) DrIFT-PHoENIX atmosphere in radiative-convective equilibrium and a temperature inversion region representing a chromospheric temperature increase.

\section{Results}

In the following, we evaluate the effect of Lyman-continuum $(\mathrm{LyC})$ irradiation at $18 \mathrm{eV}$ on the ionisation in a brown dwarf atmosphere in the three different scenarios (i)-(iii) as introduced in Sect. 2. Case (ii) and (iii) are studied in detail with respect to the effect of LyC irradiation on the electrostatic character and regarding the potential magnetic coupling of the atmosphere. We compare the effect of LyC to our previous reference results for local thermal ionisation (Rodríguez-Barrera et al. 2015). Case (i) is only briefly summarised in Sect. 3.1 and then used as a lower-limit reference for cases (ii) and (iii). Our analysis is presented for each plasma and magnetic parameter separately to allow a comparison between the different irradiating environments (Sects. 3.2-3.4).

\subsection{Effect of the interstellar radiation field}

The interstellar radiation field (ISRF) present in the ISM is a lower limit for high-energy irradiation of an old brown dwarf without a companion (free-floating objects). It has a flux $\sim 10^{7}$ photons s${ }^{-1} \mathrm{~cm}^{-2}$ (Reynolds 1984; and Table 1 ). The interstellar radiation field ionises the uppermost atmosphere layers of an ultracool objects (dashed lines in Fig. 2) considerably more than thermal ionisation (asterisks). The upper atmosphere layers reach a degree of ionisation $f_{\mathrm{e}} \sim 10^{-2}\left(f_{\mathrm{e}}=n_{\mathrm{e}} /\left(n_{\mathrm{e}}+n_{\text {gas }}\right)\right.$, $n_{\text {gas }}\left(\mathrm{cm}^{-3}\right)$ - gas density, $n_{\mathrm{e}}\left(\mathrm{cm}^{-3}\right)-$ local electron number density). The inwards-increasing gas density rapidly decreases the influence of the LyC from the ISRF on the local degree of ionisation.

\subsection{Effect of Lyman-continuum irradiation on the atmospheric electron budget}

Figure 2 shows that the LyC irradiation reaches a similar atmospheric depth and therefore ionises a similar portion of the atmosphere of a brown dwarf, independent of the incident LyC flux arriving from different radiation sources (Table 1). In the case of a young brown dwarf $(\log (g)=3.0)$, the atmospheric portion ionised by the LyC photons has a geometric extension of up to $5000 \mathrm{~km}$, for an older brown dwarf $(\log (g)=5.0)$ it affects only the upper $50 \mathrm{~km}$ as the atmosphere is far more compact. These geometrical extensions reach $P_{\text {gas }}=0.1 \ldots 10$ bar in the coolest examples studied here $\left(T_{\text {eff }}=1000 \mathrm{~K}\right)$, but remain in the low-pressure regime for the hottest atmosphere examples we studied $\left(T_{\text {eff }}=2800 \mathrm{~K}\right.$; see Fig. A. 1 for $\left(P_{\text {gas }}-z\right)$ correlation). The degree to which the gas is ionised depends on the number of interacting photons, hence on the environment in which the brown dwarf resides.
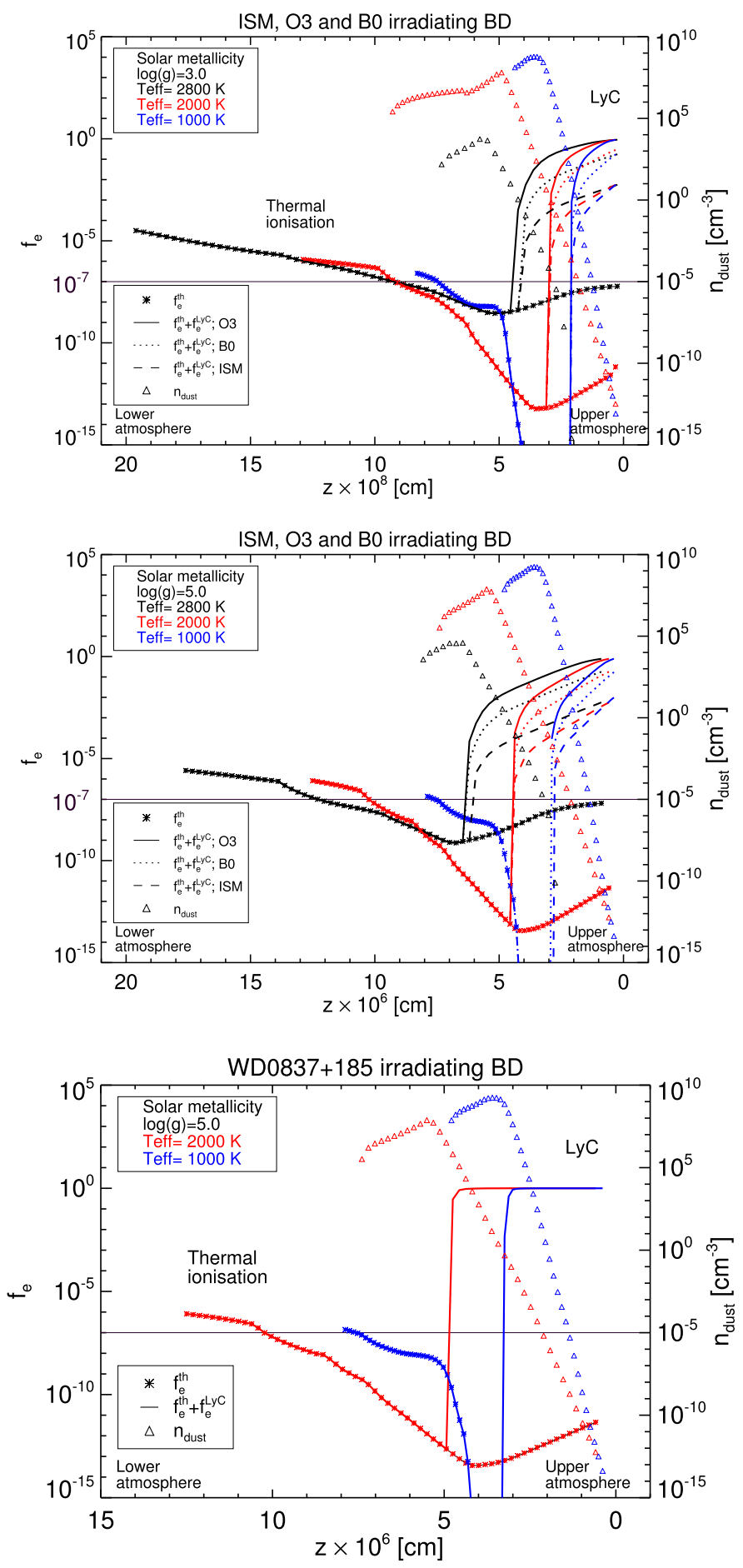

Fig. 2. Effect of Lyman-continuum irradiation from an $\mathrm{O} 3$ and a B0 star at a distance of $0.5 \mathrm{pc}$ and from the ISRF compared to the effect of the thermal ionisation on giant gas planet or young brown dwarf atmospheres (top panel), and on old brown dwarf atmospheres (middle panel). The threshold $f_{\mathrm{e}}>10^{-7}$ (thin solid black line) marks the point above which the atmospheric gas becomes partially ionised. The cloud particle number density, $n_{\text {dust }}\left(\mathrm{cm}^{-3}\right)$, is plotted as comparison to where the cloud layer is located relative to the LyC gas ionisation depth.

Star-forming region (case ii). The electron density from the photoionisation through the irradiation of an $\mathrm{O} 3$ star is three orders of magnitude higher than that from the ISRF in brown dwarf atmospheres with $\log (g)=3.0$ and two orders of magnitude higher on brown dwarf atmospheres with $\log (g)=5.0$. However, 
type B0 stars produce one and two orders of magnitude more electrons than the effect of the irradiation from the ISRF for brown dwarfs $(\log (g)=3.0,5.0)$.

Figure 2 shows that Lyman-continuum irradiation provides an efficient ionisation mechanism for the uppermost atmospheric layers in ultra-cool objects. The threshold $f_{\mathrm{e}}>10^{-7}$ marks the point above which the atmospheric gas becomes partially ionised and where plasma behaviour may emerge (Rodríguez-Barrera et al. 2015). In the case of O stars, a shell of fully ionised gas forms in the uppermost atmosphere. This ionisation effect cannot penetrate below a certain atmospheric depth, and other mechanisms may be needed to ionise the gas. For example, thermal ionisation (asterisks) starts to dominate the ionisation of the atmosphere at greater geometrical depth (i.e. higher pressures).

Figure 2 also depicts the location of the cloud layer (open triangles) that results from our DRIFT-PHOENIX atmosphere simulations. Only the outermost cloud layer would be affected by the LyC, either by the high-energy photon directly interacting with the cloud particles, or by the ionised gas depositing on the cloud particle surface. Neither of these processes are considered here, but they can cause an ionisation of the cloud particles similar to the electrification of the Moon surface dust (Helling et al. 2016a) as long as the cloud particles remain stable against electrostatic disruption (Stark et al. 2015; Helling et al. 2016c).

White dwarf - brown dwarf binary (case iii). Figure 2 (bottom panel) shows the degree of gas ionisation produced by thermal ionisation and resulting from the white dwarf LyC flux that impacts the brown dwarf atmosphere. Figure 2 (bottom panel) suggests that the predominant ionisation process is Lymancontinuum irradiation in the upper $30-50 \mathrm{~km}$ in the atmosphere of an old brown dwarf and that it ionises the upper atmosphere completely for $T_{\text {eff }}=2000-1000 \mathrm{~K}$. The uppermost layers of the cloud where the smallest particles reside (haze layer; see e.g. Helling et al. 2016b) are affected by the LyC irradiation here as well.

\subsection{Plasma parameters}

If $\omega_{\mathrm{pe}} \gg v_{\mathrm{ne}}\left(\omega_{\mathrm{pe}}-\right.$ plasma frequency, $v_{\mathrm{ne}}-$ collisional frequency neutrals-electrons; both in $\left[\mathrm{s}^{-1}\right]$ ), then electromagnetic interactions dominate electron-neutral interactions in a gas. The plasma frequency is defined as

$\omega_{\mathrm{pe}}=\left(\frac{n_{\mathrm{e}} e^{2}}{\epsilon_{0} m_{\mathrm{e}}}\right)^{1 / 2}$,

with $e$ is the electron charge [C] and $m_{\mathrm{e}}$ is the electron mass $(\mathrm{kg})$, and the collision frequency for neutral particles with electrons is

$\nu_{\mathrm{ne}}=\sigma_{\mathrm{gas}} n_{\mathrm{gas}} v_{\mathrm{th}, \mathrm{e}}$,

where $v_{\text {th.e }}$ is the thermal velocity of electrons $\left(v_{\text {th,e }}=\right.$ $\left.\left[k_{\mathrm{B}} T_{\mathrm{s}} / m_{\mathrm{s}}\right]^{1 / 2}\left[\mathrm{~m} \mathrm{~s}^{-1}\right]\right)$, and $\sigma_{\mathrm{gas}}$ is the scattering cross section of particles $\left(\sigma_{\mathrm{gas}}=\pi r_{\mathrm{gas}}^{2}=8.8 \times 10^{-17} \mathrm{~cm}^{2} ; r_{\mathrm{gas}} \approx r_{\mathrm{H}^{+}}=\right.$ $\left.5.3 \times 10^{-9} \mathrm{~cm}\right)$. Figure 3 shows where in the atmosphere the gas can be treated as a plasma. This study demonstrates that Lyman-continuum irradiation increases the atmospheric volume where the collective long-range electromagnetic interactions dominate compared to our reference study (Rodríguez-Barrera et al. 2015).
Star-forming region (case ii). Figure 3 (top and middle panels) shows that electromagnetic interactions dominate collisional processes (i.e. $\omega_{\text {pe }} / v_{\text {ne }} \gg 1$ ) for all models considered because of external LyC radiation. This is different to the case when only thermal ionisation (solid and dotted lines) is present. The effect of external irradiation in the form of LyC allows for a considerably larger atmospheric volume where electromagnetic interactions dominate compared to thermal ionisation alone. Figure 3 shows that different $T_{\text {eff }}$ and $\log (g)$ result in a difference of at least one order of magnitude (or more) in the effect on the atmosphere. All atmosphere cases appear rather similar in the very top layers with respect to their capability for electromagnetic interactions.

White dwarf - brown dwarf binary (case iii). Figure 3 (bottom panel) shows that the collective long-range electromagnetic interactions dominate the collisions between electron and neutral particles at $p_{\text {gas }} \leq 10^{-4}$ bar for the atmosphere with $T_{\text {eff }}=$ $2000 \mathrm{~K}$ and at $p_{\text {gas }} \leq 10^{-2}$ bar for the coldest atmosphere with $T_{\text {eff }}=1000 \mathrm{~K}$. The comparison of these results with the effect of the thermal ionisation shows that Lyman-continuum irradiation increases the atmospheric volume where the collective long-range electromagnetic interactions dominate by six orders of magnitude in pressure, which means that the affected region increases substantially. The LyC irradiation from a white dwarf causes a larger volume of the brown dwarf atmosphere to form a plasma, and it causes a stronger electromagnetic interaction compared to LyC from $\mathrm{O}$ and $\mathrm{B}$ stars in star-forming regions (case ii) for a given atmosphere structure (compare panels in Fig. 3).

\subsection{Magnetic parameters}

We have quantified the degree of ionisation and the plasma frequency and how they are affected by the Lyman-continuum irradiation in comparison to thermal ionisation in a substellar atmosphere for different Galactic environments. In this section, we show if and how the magnetic coupling changes as a result of external LyC photoionisation. The atmospheres are analysed with respect to a critical magnetic flux and the classical Reynolds number.

A gas is magnetised if $\omega_{\mathrm{c}, \mathrm{s}} \gg \nu_{\mathrm{n}, \mathrm{s}}\left(\omega_{\mathrm{c}, \mathrm{s}}-\right.$ cyclotron frequency, $v_{\mathrm{n}, \mathrm{s}}$ - collisional frequency, both $\left.\left[\mathrm{s}^{-1}\right]\right)$. A critical magnetic flux, $B_{\mathrm{s}}$, can be derived that is required to ensure $\omega_{\mathrm{c}, \mathrm{s}} / \nu_{\mathrm{n}, \mathrm{s}} \gg 1$ (Sect. 5.1 in Rodríguez-Barrera et al. 2015):

$B_{\mathrm{s}}=\frac{m_{\mathrm{s}}}{e} \sigma_{\text {gas, }} n_{\text {gas }}\left(\frac{k_{\mathrm{B}} T_{\mathrm{s}}}{m_{\mathrm{s}}}\right)^{1 / 2}[T]$,

where $\sigma_{\text {gas }}=\pi \times r_{\text {gas }}^{2}\left(\mathrm{~m}^{2}\right)$ is the collision, or scattering, cross section, $\mathrm{m}_{\mathrm{s}}$ is the mass of the species $s$ in $(\mathrm{kg})$, and $T_{\mathrm{s}}$ is the temperature of the species in $[\mathrm{K}] . T_{\mathrm{s}}=T_{\mathrm{gas}}$ is assumed. Hence, if a local magnetic field $B \gg B_{\mathrm{s}}$, then magnetic coupling will be possible.

The magnetic Reynolds number, $R_{\mathrm{m}}$, provides a measurement of the diffusivity of the magnetic field in a given atmospheric gas. The magnetic Reynolds number can be calculated by $R_{\mathrm{m}}=v L / \eta$, where $L(\mathrm{~m})$ is the pressure scale length of the considered plasma and $\eta\left(\mathrm{m}^{2} \mathrm{~s}^{-1}\right)$ is the diffusion coefficient. The pressure length scale is assumed to be $L=10^{3} \mathrm{~m}$, which is a representative scale height value for a brown dwarf with $\log (g)=5$ (Helling et al. 2011). The diffusion coefficient, $\eta$, can be approximated by $\eta \approx \eta_{\mathrm{d}}$ (Rodríguez-Barrera et al. 2015), 

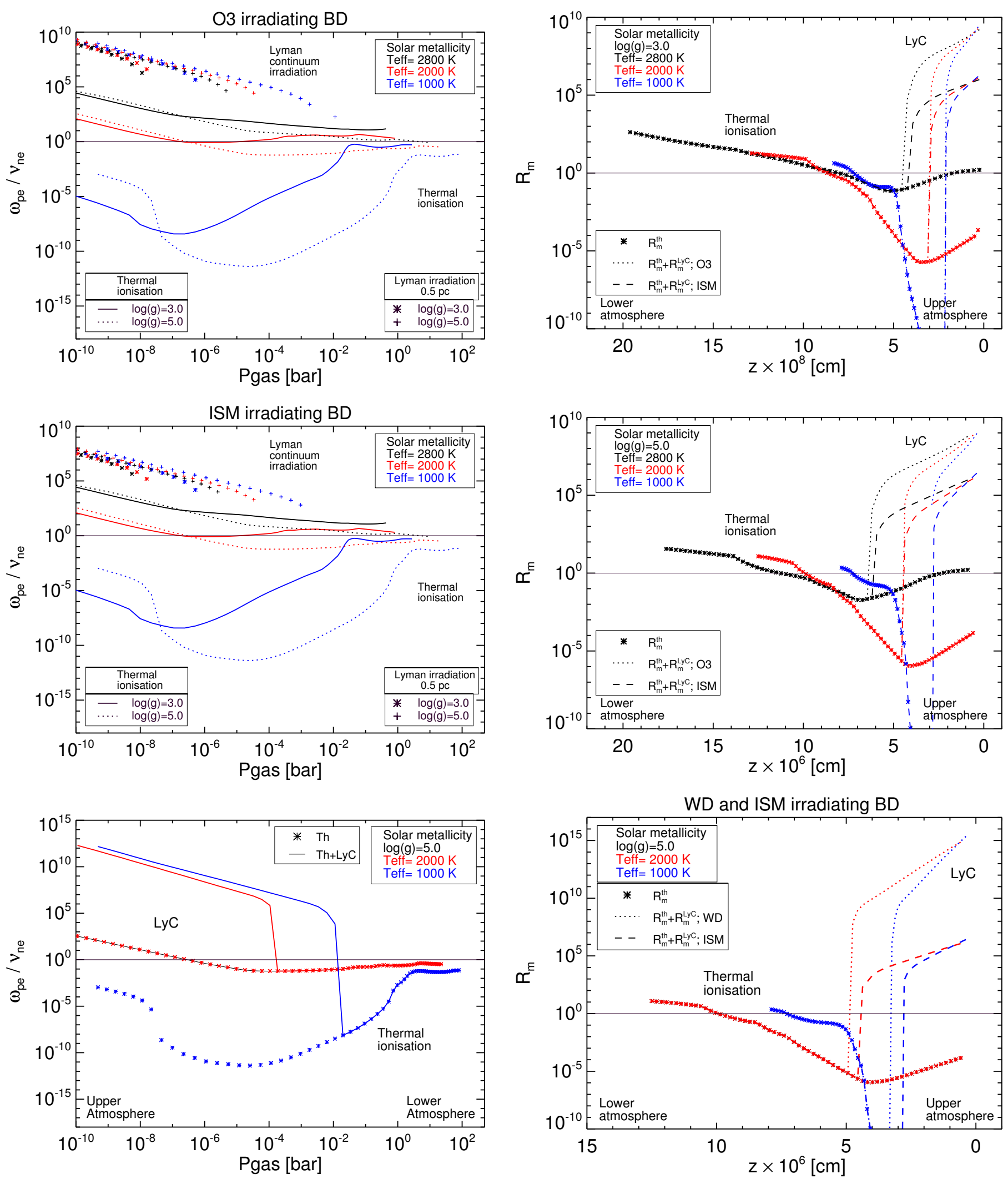

Fig. 3. Strength of electromagnetic interactions $\left(\omega_{\mathrm{pe}} / v_{\mathrm{ne}}\right)$ as a result of LyC irradiation compared to thermal ionisation in brown dwarf atmospheres in different Galactic environments. Top: (case ii) O3 star at a distance of $0.5 \mathrm{pc}$ (star-forming region). Middle: (case i) ISRF. Bottom: (case ii) white dwarf - brown dwarf binary system such as WD0837+185 with $d=0.006$ AU. Young brown dwarf atmospheres $(\log (g)=3.0)$ and old brown dwarf atmospheres $(\log (g)=5.0)$ are

Fig. 4. Magnetic Reynolds number, $R_{\mathrm{m}}\left(v_{\text {flow }}=10^{6} \mathrm{~cm} \mathrm{~s}^{-1}\right)$ as result of LyC irradiation compared to thermal ionisation in different Galactic environments: Top: young brown dwarfs in a star-forming region (O3 star, $d=0.5 \mathrm{pc}$ ). Middle: old brown dwarf in a star-forming region (O3 star, $d=0.5 \mathrm{pc}$ ). Bottom: old brown dwarf in a white dwarf binary system such as WD0837+185 with $d=0.006 \mathrm{AU}$. 
where $\eta_{\mathrm{d}}$ is the decoupled diffusion coefficient $\eta_{\mathrm{d}}=c^{2} v_{\mathrm{ne}} / \omega_{\mathrm{pe}}^{2}$. The plasma has reached the ideal MHD regime if $R_{\mathrm{m}} \gg 1$ and the coupling between the plasma and the background magnetic field is complete.

Star-forming region (case ii). Figure 5 shows where electrons resulting from LyC irradiation from an $\mathrm{O}$ star can be magnetised in an M dwarf/browndwarf/planet atmosphere compared to thermal ionisation, that is, where $B \gg B_{\mathrm{s}}$. We furthermore observe that

- for atmospheres with $T_{\text {eff }}=2800 \mathrm{~K}$ (M dwarfs and young brown dwarfs), a typical background magnetic field flux of $B=10^{3} \mathrm{G}$ magnetises the ambient electrons at $p_{\text {gas }}<5$ bar,

- for atmospheres with $T_{\text {eff }}=1000 \mathrm{~K}$ (planets), a typical background magnetic field flux of $B=10 \mathrm{G}$ magnetises the electrons at $p_{\text {gas }}<5 \times 10^{-2}$ bar.

Figure 4 shows the magnetic Reynolds number as the result of the thermal ionisation plus the photoionisation by Lymancontinuum irradiation from an $\mathrm{O}$ star and from the ISRF. The results from Fig. 4 suggest that

- a decrease in $T_{\text {eff }}$, for a given $\log (g)$ and $[\mathrm{M} / \mathrm{H}]$, decreases the atmospheric volume where $R_{\mathrm{m}} \gg 1$,

- an ideal MHD be haviour $\left(R_{\mathrm{m}} \gg 1\right)$ can be considered in the upper atmosphere owing to the significant increase in electron density $\left(R_{\mathrm{m}} \propto n_{\mathrm{e}}\right)$ by the effect of the Lyman-continuum irradiation compared to the effect of thermal ionisation alone.

White dwarf - brown dwarf binary (case iii). Figure 5 (bottom) shows that LyC irradiation from white dwarfs would not only magnetise electrons in brown dwarf atmospheres, but also in $\mathbf{M}$ dwarf and giant gas planet atmospheres:

- for atmospheres with $T_{\text {eff }}=2000 \mathrm{~K}$ (brown dwarfs and $\mathrm{M}$ dwarfs) with a background magnetic field flux of $B=10^{3} \mathrm{G}$, electrons are magnetised for $p_{\text {gas }}<5$ bar,

- for atmospheres with $T_{\text {eff }}=1000 \mathrm{~K}$ (giant gas planets), a background magnetic field flux of $B=10 \mathrm{G}$ can magnetise the electrons at $p_{\text {gas }}<5 \times 10^{-2}$ bar.

Figure 4 (bottom panel) shows the magnetic Reynolds number for LyC irradiation from a WD and the ISM compared to the effect of thermal ionisation. The results are similar to case (ii), except that the Reynolds number reaches higher values if the brown dwarf were affected by LyC from a white dwarf.

\subsection{Free-free emission luminosity from strongly ionised, optically thin atmospheres}

When we assume that these uppermost highly ionised atmosphere layers are optically thin, we can estimate the resulting luminosity from free-free emission (Eq. (2)) for the three cases of Galactic environment considered here. The emissivity $\epsilon^{\mathrm{ff}} \sim T_{\mathrm{em}}^{1 / 2} n_{\mathrm{e}} n_{\mathrm{i}} \times f\left(\mathrm{e}^{1 / T_{\mathrm{em}}}\right)($ Eq. (3)) requires information about the local densities, $n_{\mathrm{e}}, n_{\mathrm{i}}$, and the temperature of the emitting gas. The local electron number densities are a direct result of our calculation. For the local electron temperature, $T_{\mathrm{em}}[\mathrm{K}]$, we tested two cases: $T_{\mathrm{em}}=T_{\text {gas }}$ with $T_{\text {gas }}$ the gas temperature as result of the Drift-Phoenix model atmosphere. This case assumes that no effective heating of the ionised gas occurs. In the second case, we used $T_{\text {em }}=T_{\text {chrom }}$ with $T_{\text {chrom }}=$ const, a preset temperature of expected chromospheric values, representing potential heating through photoionisation and mechanical waves. We have no information about chromospheric temperatures on brown dwarfs, and suggestions in the literature vary:
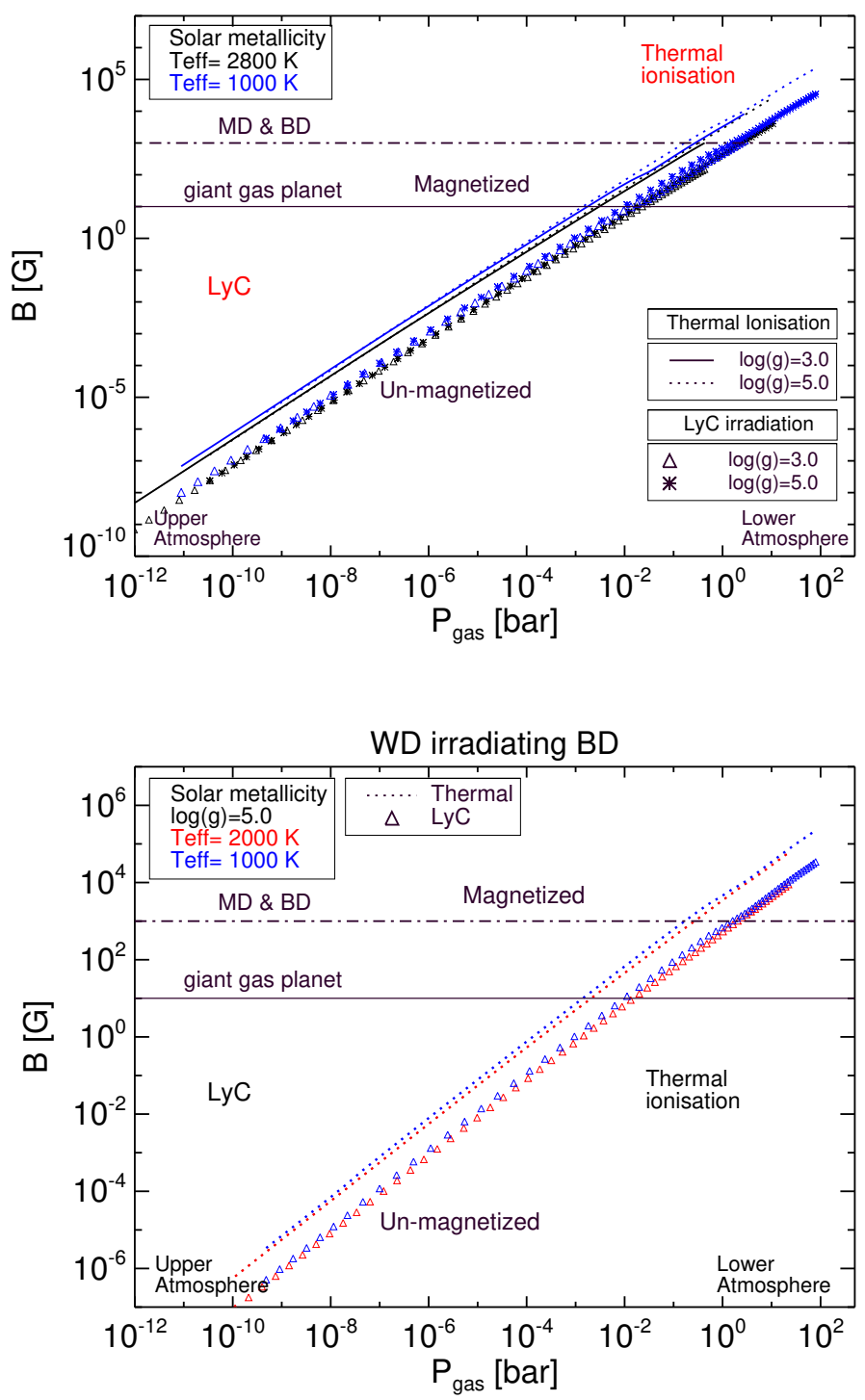

Fig. 5. Magnetic flux density, $B=B_{\mathrm{s}}$, required for the electrons to achieve magnetic coupling in ultra-cool objects. The effect of Lymancontinuum irradiation is compared to the effect of thermal ionisation (the solid line depicts $\log (g)=3.0$, and the dotted line shows $\log (g)=$ 5.0). Top: brown dwarfs irradiated from an $\mathrm{O} 3$ star from a distance of $0.5 \mathrm{pc}$ in a star-forming region (asterisks - late-M dwarfs and young brown dwarf atmospheres, $\log (g)=3.0$; the triangle - old brown dwarfs, $\log (g)=5.0)$. Bottom: binary system with a WD star irradiating a brown dwarf from a distance of $d=0.006 \mathrm{AU}$ (blue triangle - cold, old brown dwarf, red triangle - warm, old brown dwarf).

Burgasser et al. (2013) suggested that the brown dwarf 2MASS J13153094-2649513 (L7) emits electrons with temperature of $T_{\mathrm{em}} \approx 10^{9}-10^{10} \mathrm{~K}$, Osten et al. (2009) quoted a temperature of $T_{\mathrm{em}} \approx 10^{6} \mathrm{~K}$, and Schmidt et al. (2015) applied $T_{\mathrm{em}}=10^{4}$ $\mathrm{K}$. In order to provide first estimates based on our ionisation calculations, we followed the examples by Fuhrmeister et al. (2010) and Schmidt et al. (2015) in postulating an isothermal temperature layer with temperatures higher than in the inner atmosphere. Such a layer could also be the result of MHD wave heating as a result of the magnetic coupling shown in Fig. 5. The temperature of the emitting electrons may also decouple from the gas because their mass is significantly lower than that of neutrals and ions. Hence, because the electrons will lose a smaller portion of their energy during a collision, they may not be in thermal 
Table 2. Upper limits for X-ray luminosity for $0.5-8 \mathrm{KeV}$ from free-free emission, $L_{\mathrm{tot}}^{\mathrm{ff}}\left[\mathrm{erg} \mathrm{s}^{-1}\right]$, for optically thin, ionised gases.

\begin{tabular}{|c|c|c|c|c|c|}
\hline \multirow{2}{*}{ Source } & \multirow{2}{*}{$T_{\text {eff }}[\mathrm{K}]$} & \multicolumn{2}{|c|}{$L_{\mathrm{tot}}^{\mathrm{ff}}\left[\mathrm{erg} \mathrm{s}^{-1}\right]$ for $T_{\mathrm{em}}=10^{5} \mathrm{~K}$} & \multicolumn{2}{|c|}{$L_{\mathrm{tot}}^{\mathrm{ff}}\left[\mathrm{erg} \mathrm{s}^{-1}\right]$ for $T_{\mathrm{em}}=10^{6} \mathrm{~K}$} \\
\hline & & $\log (g)=3.0$ & $\log (g)=5.0$ & $\log (g)=3.0$ & $\log (g)=5.0$ \\
\hline \multirow[t]{3}{*}{$\mathrm{O} 3$} & 2800 & $1.8 \times 10^{+2}$ & $8.5 \times 10^{+1}$ & $7.0 \times 10^{+27}$ & $8.6 \times 10^{+26}$ \\
\hline & 2000 & $3.4 \times 10^{+3}$ & $7.4 \times 10^{+3}$ & $3.4 \times 10^{+28}$ & $7.5 \times 10^{+28}$ \\
\hline & 1000 & $1.6 \times 10^{+6}$ & $1.2 \times 10^{+7}$ & $1.6 \times 10^{+31}$ & $1.2 \times 10^{+32}$ \\
\hline \multirow[t]{3}{*}{ B0 } & 2800 & 2.1 & 1.2 & $2.1 \times 10^{+25}$ & $1.2 \times 10^{+25}$ \\
\hline & 2000 & $3.5 \times 10^{+1}$ & $1.0 \times 10^{+2}$ & $3.6 \times 10^{+26}$ & $1.0 \times 10^{+27}$ \\
\hline & 1000 & $5.7 \times 10^{+3}$ & $1.6 \times 10^{+5}$ & $5.7 \times 10^{+28}$ & $1.6 \times 10^{+30}$ \\
\hline \multirow[t]{3}{*}{ ISM } & 2800 & $1.8 \times 10^{-3}$ & $1.0 \times 10^{-3}$ & $1.8 \times 10^{+22}$ & $1.0 \times 10^{+22}$ \\
\hline & 2000 & $1.0 \times 10^{-2}$ & $8.9 \times 10^{-2}$ & $1.0 \times 10^{+23}$ & $9.0 \times 10^{+23}$ \\
\hline & 1000 & 5.2 & $1.4 \times 10^{+2}$ & $5.3 \times 10^{+25}$ & $4.0 \times 10^{+32}$ \\
\hline \multirow[t]{2}{*}{ WD } & 2000 & - & $8.3 \times 10^{+12}$ & - & $8.4 \times 10^{+37}$ \\
\hline & 1000 & - & $2.5 \times 10^{+16}$ & - & $2.6 \times 10^{+41}$ \\
\hline
\end{tabular}

Notes. We assumed ultra-cool objects $\left(T_{\text {eff }}=2800 \mathrm{~K}, 2000 \mathrm{~K}, 1000 \mathrm{~K} ; \log (g)=3.0,5.0\right)$ in case (ii) irradiated by an O3 or B0 star at distance $d=0.5 \mathrm{pc}$, in case (iii) irradiated by a WD star at distance $d=0.006 \mathrm{AU}$, and in case (i) from the ISRF. We neglected any shadowing effects. The temperature of the emitting gas was set to $T_{\mathrm{em}}=10^{5} \mathrm{~K}$ and $T_{\mathrm{em}}=10^{6} \mathrm{~K}$ to mimic a chromospheric temperature or an otherwise thermally decoupled emitting electron population.

equilibrium with their surrounding in regions from which $\mathrm{X}$-ray and radio emission emerge. We tested two values, $T_{\mathrm{em}}=10^{5} \mathrm{~K}$ and $T_{\mathrm{em}}=10^{6} \mathrm{~K}$.

Table 2 summarises the free-free emission luminosities, $L_{\mathrm{tot}}^{\mathrm{ff}}$, that would occur if the LyC ionised gas were optically thin and had the temperature $T_{\mathrm{em}}$. The highest amount of free-free emission luminosity is to be expected from brown dwarfs orbiting white dwarfs, the lowest from brown dwarfs in the interstellar medium. The strong increase of the values in Table 2 when we increased the local gas temperature to $10^{6} \mathrm{~K}$ results from the steepness of the high-energy tail of the Planck function.

Star-forming region (case ii). The free-free emission luminosities in the X-ray energy interval $0.5-8 \mathrm{KeV}$ in Table 2 reach values that are similar to observations from non-accreting brown dwarfs at X-ray wavelengths (Sicilia-Aguilar et al. 2008; Kashyap et al. 2008 and Feigelson et al. 2003) if the upper LyC ionised atmosphere can be heated substantially. The emissivity is very low if the emitting part of the atmosphere would emit with the local LTE gas temperature (Fig. 1). Schmidt et al. (2015) presented the $H_{\alpha}$ emission of ultra-cool (M7-L8) brown dwarfs and suggested that these observations required the presence of a chromosphere. Forbrich \& Preibisch (2007) suggested that the candidate brown dwarf B185839.6-365823, with $\log L_{\mathrm{x}}$ $=28.43\left(L_{\mathrm{x}} \sim 2.7 \times 10^{28} \mathrm{erg} \mathrm{s}^{-1}\right)$, required a high plasma temperature in order to fit the observed spectrum. Another example for a very late dwarf, the M8.5 brown dwarf B185831.1-370456, was observed with a luminosity from free-free emission of $\log L_{\mathrm{x}}$ $=26.9\left(L_{\mathrm{x}} \sim 8 \times 10^{26} \mathrm{erg} \mathrm{s}^{-1}\right)$. Neuhäuser et al. (1999) reported a $\sim 1$ Myr old brown dwarf (Cha $\left.H_{\alpha} 1\right)$ with a quiescent emission at X-ray wavelengths of $\log L_{\mathrm{x}}=28\left[\mathrm{erg} \mathrm{s}^{-1}\right]$. They suggested that magnetic activity must be present to explain these observations. Feigelson et al. (2003) detected 525 objects in a massive cluster (Orion nebula) at energies of $0.5-8 \mathrm{KeV}$ (X-rays); 144 of these objects are catalogued as brown dwarfs $\left(\log M \leq-0.2 M_{\odot}\right)$ (their Table 1) with an average of the X-ray emitted luminosity of $\log L_{\mathrm{x}} \approx 28.84 \mathrm{erg} \mathrm{s}^{-1}$. Our estimates of the free-free luminosity that is emitted from brown dwarfs that form a shell of highly ionised but optically thin gas in star-forming regions are of the same order of magnitude. Our estimates, which are summarised in Table 2, show that the resulting luminosity from free-free emission in the energy interval $0.5-8 \mathrm{KeV}$ increases if - the object forms a chromosphere with a substantially increased temperature compared to LTE values.

- the $T_{\text {eff }}$ decreases.

An increase in surface gravity, hence a decrease in atmospheric scale heights, does lead to an increased luminosity in most but not all cases.

White dwarf - brown dwarf binary (case iii). Table 2 also presents the resulting luminosity from free-free emission (thermal Bremsstrahlung irradiation) of a brown dwarf that is ionised by Lyman-continuum irradiation from a WD star. The results show that $L_{\text {tot }}^{\mathrm{ff}}$ is higher in a white dwarf - brown dwarf system than in a star-forming region or through the ISRF because of the close orbital separation between the white dwarf and the brown dwarf. Recent optical and near-infrared observations of the close white dwarf - brown dwarf non-interacting binary system WD0137-349 by Longstaff et al. (2017) showed that He, $\mathrm{Na}, \mathrm{Mg}, \mathrm{Si}, \mathrm{K}, \mathrm{Ca}, \mathrm{Ti}$, and $\mathrm{Fe}$ lines were emitted from the brown dwarf companion. This is a strong indication for a temperature inversion in the upper atmosphere, and in combination with the detected $\mathrm{H} \alpha$ emission, it is a strong indication that a high-temperature plasma is present in this cool companion to a white dwarf. This conclusions for such systems is supported by Casewell et al. (2018), who detected Mg I and Ca II emission lines in addition to $\mathrm{H} \alpha$ lines in the (shortest-period) noninteracting, white dwarf - brown dwarf post-common-envelope binary known as EPIC 21223532.

\section{Discussion}

Unexpectedly powerful emissions at radio, X-ray, and $H_{\alpha}$ wavelengths from very low-mass objects (ultra-cool dwarfs) have been observed by different groups (e.g. Williams et al. 2014; Burgasser et al. 2013; Berger 2002; Route \& Wolszczan 2012 and see Pineda et al. 2017 for a recent survey). Sorahana et al. (2014) suggested that weakened $\mathrm{H}_{2} \mathrm{O}(2.7 \mu \mathrm{m}), \mathrm{CH}_{4}(3.3 \mu \mathrm{m})$, and $\mathrm{CO}(4.6 \mu \mathrm{m})$ absorption in combination with moderate $\mathrm{H} \alpha$ emission could be linked to chromospheric activity and its 
effect on the underlaying atmosphere structure. Observations by Schmidt et al. (2016, 2015) support this interpretation. They postulated the presence of a chromosphere to reproduce extensive $\mathrm{H} \alpha$-activity survey data for brown dwarfs. Schmidt et al. (2016) reported an old L0 dwarf emitting powerful emissions at $H_{\alpha}$ and near-infrared wavelengths. Such powerful emissions suggest that magnetic activity must be present even in old-type brown dwarfs, and it implies the presence of a magnetised atmospheric plasma even in such ultra-cool objects. However, no consistent MHD simulations for brown dwarfs have been carried out so far. Wedemeyer et al. (2013) studied the formation of a chromosphere on fully ionised M dwarfs. Non-ideal (i.e. partially ionised) MHD simulations by Tanaka et al. (2015) suggested the formation of a temperature inversion by magneto-convection processes and Alfvén-wave heating (see also Brady \& Arber 2016; Mullan \& MacDonald 2016 and Reep \& Russell 2016) in giant gas planets. According to our work, this might also work for brown dwarfs and lead to the formation of a chromosphere.

The radio emission from brown dwarfs has also been examined by Nichols et al. (2012), for instance, who studied the properties of radio emissions in ultra-cool dwarfs assuming the occurrence of auroral regions. They suggested that the coupling between the atmospheric plasma and the magnetic field causes a (postulated) high-latitude ionosphere and generates auroral processes. A loss of particles to the ionosphere was suggested. Speirs et al. (2014) presented a theoretical approach for cyclotron radio emission from Earth's auroral region, showing that the radio emission results from a backward-wave cyclotronmaser emission process.

Pineda et al. (2017) have provided a concise overview of these different approaches, suggesting that a transition from Sunlike chromospheric processes to Jupiter-like large-scale magnetospheric current systems occurs at the low-mass end of the main sequence. Our paper has added to this discussion by addressing the question of the origin of the pool of electrons that allows a chromosphere to form or a magnetospheric current system to develop, assuming that a magnetic field is present. Although we do not present a consistent simulation to demonstrate the formation of a chromospheric region on brown dwarfs, first estimates of the X-ray luminosity of a hot, optically thin gas that is ionised by Lyman-continuum radiation suggest values that are similar to observations of non-accreting brown dwarfs in star-forming regions.

The required high electron density could affect the cloud layer in brown dwarf atmospheres. Helling et al. (2016c) suggested that cloud particles might be destroyed if the electron temperature is $>10^{5} \mathrm{~K}$. This would occur if a chromosphere-like temperature increase were to coincide with some part of the cloud layer where a strong gas ionisation occurs (only the uppermost atmosphere, Fig. 2).

\section{Conclusions}

Lyman-continuum irradiation causes a considerable increase in ionisation in the upper and outermost atmospheric regions of ultra-cool objects, forming a shell of substantial local ionisation. We showed that these atmospheric regions exhibit a far stronger plasma than can be achieved with thermal ionisation alone, and it would therefore be reasonable to call this part of the atmosphere an ionosphere. If a sufficient global magnetic field is present, magnetic coupling of these highly ionised atmospheric layers occurs, and we may therefore expect the formation of a chromosphere if the object is sufficiently convective such that
MHD heating mechanisms similar to that on M dwarfs can occur (Wedemeyer et al. 2013).

Different Galactic environments were investigated (ISRF, star-forming region, white dwarf - brown dwarf binary). Lymancontinuum radiation from the interstellar radiation field has the smallest effect on the degree of ionisation. The outer atmosphere of a brown dwarf binary as companion of a white dwarf, however, can be expected to be fully ionised. More rigorous followup simulations are therefore warranted in order to study effects such as magnetically driven mass loss or even mass transfer in WD-BD systems.

Acknowledgements. M. I. R.-B. and Ch. H. acknowledge financial support of the European Community under the FP7 by the ERC starting grant 257431. Ch. H. thanks for the hospitality of the Kaptyn Astronomical Insititut at the University of Groningen, and travel support from NWO and LKBF. We thank A. Sicilia-Aguilar and P. Rimmer for the insightful and valuable discussions of the manuscript. Most literature search was performed using the ADS. We gratefully acknowledge our local computer support.

\section{References}

Antonova, A., Hallinan, G., Doyle, J. G., et al. 2013, A\&A, 549, A131 Berger, E. 2002, ApJ, 572, 503

Brady, C. S., \& Arber, T. D. 2016, ApJ, 829, 80

Burgasser, A. J., Melis, C., Zauderer, B. A., \& Berger, E. 2013, ApJ, 762, L3

Burgasser, A. J., Melis, C., Todd, J., et al. 2015, AJ, 150, 180

Casewell, S. L., Burleigh, M. R., Wynn, G. A., et al. 2012, ApJ, 759, L34

Casewell, S. L., Burleigh, M. R., Lawrie, K. A., et al. 2013, Mem. Soc. Astron. It. 84, 1022

Casewell, S. L., Lawrie, K. A., Maxted, P. F. L., et al. 2015, MNRAS, 447, 3218

Casewell, S. L., Braker, I. P., \& Parsons, S. G. 2018, MNRAS, 476, 1405

Feigelson, E. D., Gaffney, III, J. A., Garmire, G., Hillenbrand, L. A., \& Townsley, L. 2003, ApJ, 584, 911

Forbrich, J., \& Preibisch, T. 2007, A\&A, 475, 959

Fuhrmeister, B., Schmitt, J. H. M. M., \& Hauschildt, P. H. 2005, A\&A, 439, 1137

Fuhrmeister, B., Schmitt, J. H. M. M., \& Hauschildt, P. H. 2010, A\&A, 511, A83

Gizis, J. E., Burgasser, A. J., Berger, E., et al. 2013, ApJ, 779, 172

Hallinan, G., Antonova, A., Doyle, J. G., et al. 2006, ApJ, 653, 690

Helling, Ch., \& Casewell, S. 2014, A\&ARv, 22, 80

Helling, Ch., \& Woitke, P. 2006, A\&A, 455, 325

Helling, Ch., Dehn, M., Woitke, P., \& Hauschildt, P. H. 2008a, ApJ, 675, L105 Helling, Ch., Dehn, M., Woitke, P., \& Hauschildt, P. H. 2008b, ApJ, 677, L157

Helling, C., Jardine, M., Witte, S., \& Diver, D. A. 2011, ApJ, 727, 4

Helling, Ch., Jardine, M., Diver, D., \& Witte, S. 2013, Planet. Space Sci., 77, 152

Helling, Ch., Harrison, R. G., Honary, F., et al. 2016a, Surveys Geophy., 37, 705 Helling, Ch., Lee, G., Dobbs-Dixon, I., et al. 2016b, MNRAS, 460, 855

Helling, Ch., Rimmer, P. B., Rodriguez-Barrera, I. M., et al. 2016c, Plasma Phys. Controlled Fusion, 58, 074003

Hills, J. G. 1973, A\&A, 28, 63

Kao, M. M., Hallinan, G., Pineda, J. S., et al. 2016, ApJ, 818, 24

Kashyap, V. L., Drake, J. J., \& Saar, S. H. 2008, ApJ, 687, 1339

Longstaff, E. S., Casewell, S. L., Wynn, G. A., Maxted, P. F. L., \& Helling, Ch. 2017, MNRAS, 471, 1728

Lystrup, M. B., Miller, S., Dello Russo, N., Vervack, Jr., R. J., \& Stallard, T. 2008, ApJ, 677, 790

Mohanty, S., Basri, G., Shu, F., Allard, F., \& Chabrier, G. 2002, ApJ, 571, 469

Mullan, D. J., \& MacDonald, J. 2016, ApJ, 818, 154

Neuhäuser, R., Briceño, C., Comerón, F., et al. 1999, A\&A, 343, 883

Nichols, J. D., Burleigh, M. R., Casewell, S. L., et al. 2012, ApJ, 760, 59

Osten, R. A., Phan-Bao, N., Hawley, S. L., Reid, I. N., \& Ojha, R. 2009, ApJ, 700, 1750

Osterbrock, D., \& Ferland, G. 2006, Astrophysics of Gaseous Nebulae and Active Galactic Nuclei (University Science Books)

Pineda, J. S., Hallinan, G., Kirkpatrick, J. D., et al. 2016, ApJ, 826, 73

Pineda, J. S., Hallinan, G., \& Kao, M. M. 2017, ApJ, 846, 75

Reep, J. W., \& Russell, A. J. B. 2016, ApJ, 818, L20

Reynolds, R. J. 1984, ApJ, 282, 191

Rimmer, P. B., \& Helling, Ch. 2013, ApJ, 774, 108

Rimmer, P. B., \& Helling, Ch. 2016, ApJS, 224, 9

Rimmer, P. B., Helling, Ch., \& Bilger, C. 2014, Int. J. Astrobiol., 13, 173 
Rodríguez-Barrera, M. I., Helling, Ch., Stark, C. R., \& Rice, A. M. 2015, MNRAS, 454, 3977

Route, M., \& Wolszczan, A. 2012, ApJ, 747, L22

Schmidt, S. J., Hawley, S. L., West, A. A., et al. 2015, AJ, 149, 158

Schmidt, S. J., Shappee, B. J., Gagné, J., et al. 2016, ApJ, 828, L22

Sicilia-Aguilar, A., Henning, T., Juhász, A., et al. 2008, ApJ, 687, 1145

Sorahana, S., Suzuki, T. K., \& Yamamura, I. 2014, MNRAS, 440, 3675

Speirs, D. C., Bingham, R., Cairns, R. A., et al. 2014, Phys. Rev. Lett., 113, 155002

Stark, C. R., Helling, Ch., \& Diver, D. A. 2015, A\&A, 579, A41

Steele, P. R., Saglia, R. P., Burleigh, M. R., et al. 2013, MNRAS, 429, 3492

Stelzer, B. 2004, ApJ, 615, L153

\section{Appendix A: Supplementary details}

This appendix contains supplementary information about the relation between the local gas pressure, $P_{\text {gas }}$, and the geometrical extension of the atmospheres, $z$, in Fig. A.1.
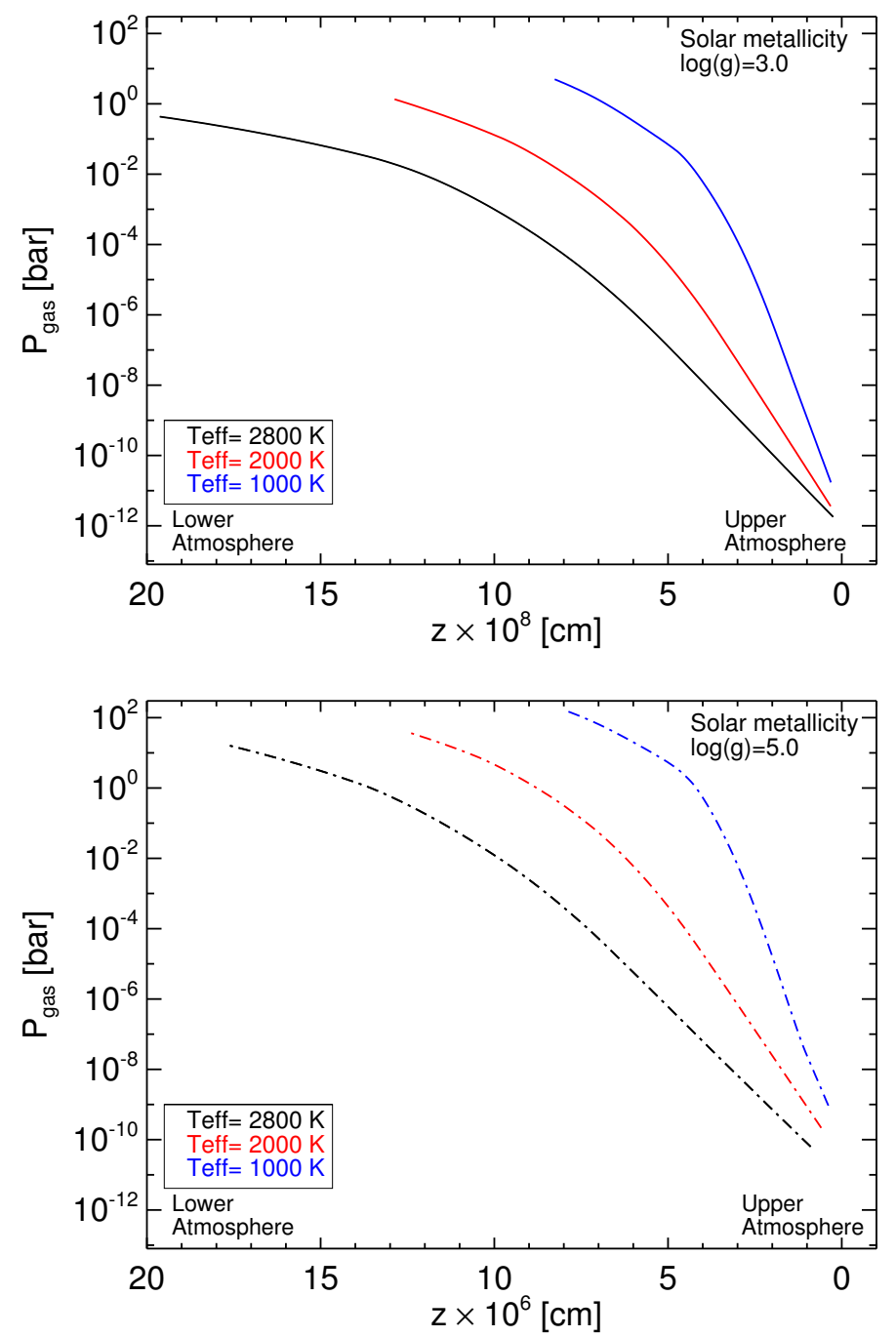

Fig. A.1. Local gas pressure, $P_{\text {gas }}$ as a function of vertical geometrical extension of the atmosphere, $z(\mathrm{~cm})$ given by Drift-PhoENIX. Top: young brown dwarf atmospheres. Bottom: old brown dwarf atmospheres.
Sternberg, A., Hoffmann, T. L., \& Pauldrach, A. W. A. 2003, ApJ, 599, 1333

Tanaka, Y. A., Suzuki, T. K., \& Inutsuka, S.-I. 2015, ApJ, 809, 125

Wedemeyer, S., Ludwig, H.-G., \& Steiner, O. 2013, Astron. Nachr., 334, 137

Williams, P. K. G., Berger, E., Irwin, J., Berta-Thompson, Z. K., \& Charbonneau, D. 2014, ApJ, 799, 192

Witte, S., Helling, Ch., \& Hauschildt, P. H. 2009, A\&A, 506, 1367

Witte, S., Helling, Ch., Barman, T., Heidrich, N., \& Hauschildt, P. H. 2011, A\&A, 529, A44

Woitke, P., \& Helling, Ch. 2004, A\&A, 414, 335

Wood, K., \& Loeb, A. 2000, ApJ, 545, 86

Wood, K., Mathis, J. S., \& Ercolano, B. 2004, MNRAS, 348, 1337 